\title{
$\$$ Research Square

\section{KLF5-mediated Eppk1 expression promotes cell proliferation in cervical cancer via the p38 signaling pathway}

\section{Zhe Pan}

North China University of Science and Technology

Xiao Liu

Hebei Medical University Fourth Affiliated Hospital and Hebei Provincial Tumor Hospital

Quan Chang

North China University of Science and Technology

Jin-jin Zhang

North China University of Science and Technology

Na Hua

Tangshan constoms People's republic of China

Guo-Hua Li

Department of Oncology, the Second Hospital of Tangshan

Dong Ma ( $\triangle$ mamamadong@163.com )

North China University of Science and Technology

Research

Keywords: Cervical cancer, Eppk1, KLF5, proliferation, EGFR, p38 signal

Posted Date: May 29th, 2020

DOI: https://doi.org/10.21203/rs.3.rs-31063/v1

License: (9) This work is licensed under a Creative Commons Attribution 4.0 International License.

Read Full License 


\section{Abstract}

Background: Epiplakin1 (Eppk1) is part of the EGF signal and is involved in cytoskeleton reorganization and cell proliferation. However, the role of Eppk1 in cervical cancer remains unknown.

Objective: To determine the role of EPPK1 on cell proliferation in cervical cancer.

Methods: The expression of Eppk1 and KLF5 as well as their correlation were assessed by RNA-seq, qRTPCR, TCGA database and immunofluorescence staining. In CC cell lines, adenovirus-mediated overexpression or knockdown of KLF5 and Eppk1 as well as corresponding assessment of cell proliferation and signaling were determined by western blot and CCK8 experiments. Assays of lucifase reporter gene and CHIP were used to investigate mechanism between KLF5 and Eppk1.

Results: Eppk1 expression was markedly in CC tissues and cell lines companied by KLF5 upregulation. The results of immunofluorescence staining further showed that the increased expression of Eppk1 and KLF5 correlated with progression of cervical tumorigenesis. Overexpression of KLF5 significantly increased Eppk1 expression at transcription and translation levels. Conversely, the knockdown of KLF5 by siRNA against KLF5 decreased Eppk1 expression. Mechanical studies showed that KLF5 activated Eppk1 transcription by direct binding to the Eppk1 promoter. Gain- and loss-of-function experiments showed that KLF5 promoted cell proliferation in Hela by upregulating Eppk1 expression. Moreover, KLF5-mediated the activation of EGFR and p38 signaling significantly decreased after Eppk1 knockdown companied with reduction of proliferating activity, suggesting that Eppk1 lies upstream of p38 signaling affecting cell proliferation in CC. Finally, the expression of Eppk1 positively correlated with tumor size.

Conclusions: Eppk1 may be an effective therapeutic target on affecting EGFR-associated p38 signaling pathway and cell proliferation in cervical cancer.

\section{Background}

Cervical cancer represents a major clinical problem as it is globally the most common malignancy of the female reproductive system and usually affects young women [1]. Clinically, its late results of treatment and prognosis are still in bad, the overall five-year survival rate was only $60 \%$ [2]. The pathogenesis is still unclear, whereas persistent high-risk human papillomavirus (HPV) infection is the main cause of cervical cancer, suggesting that the investigation of prognostic markers and therapeutic targets in CC is crucial [3]. The application of high-throughput sequencing technologies, such as RNA-sequence, provides convenient means for finding them in the treatment of CC.

Epiplakin1 (Eppk1), a member of the plakin gene family, is a universal cell linker protein originally identified as a 450-kDa human epidermal self-antigen, expressed in liver, small intestine, stomach, salivary glands, esophagus and skin [4,5]. Plakin protein is known to bind cytoskeletal myofilaments and anchor at the cytoplasmic membrane junction, which is a component of EGF signaling and involved in cytoskeletal aggregation and proliferation signal transduction of tumor cells [6]. The different expression 
of Eppk1 has been reported in various cancers such as hepatocellular carcinoma [7], bladder urothelial carcinoma [8], cervical cancer [9], and Eppk1 has been studied in pancreatic cancer [10], but the role of Eppk1 in CC remains unknown.

Kruppel-like factors 5 (KLF5) exerts cell biological functions such as cell proliferation, migration and differentiation by regulating many important target genes [11]. Our previous study found that KLF5 also plays a role in cytoskeletal aggregation and proliferation, and plays an important role in the proliferation of cervical cancer [12], suggesting that whether there is a link between Eppk1 and KLF5 may be involved in the pathological progression of cervical cancer.

The present study investigated the role of Eppk1 in cervical tumogenesis and examined whether KLF5 regulating Eppk1 expression affects EFGR activation and its downstream p38 signaling, which will provide molecular targets for clinical late treatment and prognosis of cervical cancer.

\section{Materials And Methods}

\section{Clinical samples}

Selection in September 2014 to September 2017 tangshan worker hospital cases cervical biopsy and surgical treatment, clinical data integrity, and did not receive preoperative radiation and chemotherapy treatment, a total of 152 cases, of which the normal cervical 34 cases (control), cervical intraepithelial neoplasia (CIN) in 78 cases (including $\mathrm{CIN} \otimes 31$ cases, $\mathrm{CIN} \otimes-\otimes 47$ cases) and 40 cases cervical squamous cell carcinomas (SCC). The general conditions of the patients were investigated, and the age of the patients, age of menarche, number of sexual partners, number of pregnancies, number of abortions, smoking and drinking had no statistical significance $(P>0.05)$. The median age of all participants was 45 and ranged from 25 to 79. After diagnosis, they underwent surgical resection of primary cervical cancer in the Department of Obstetrics and Gynecology of Tangshan workers' hospital. The histological types and grades of tumors were classified according to WHO criteria. The stage of each cancer was determined according to the International Federation of Gynaecology and Obstetrics (FIGO 2000) criteria. According to the agency guidelines, all of these patients had obtained informed consent prior to sample collection, and the study was approved by the Ethics Committee of North China University of Technology in Tangshan, Hebei province, China.

\section{Cell culture}

Human normal cervical epithelial cell (HcerEpic) and human cervical (HeLa, SiHa and C33A) cancer cell lines were purchased from the Cell Culture Center (Manassas, VA). And containing RPMI 1640 medium containing $10 \%$ fetal bovine serum, $5 \%$ carbon dioxide, $37^{\circ} \mathrm{C}$ constant temperature incubator, logarithmic growth phase cells were used for the following experiments.

\section{Adenoviral vector, siRNA and transfection}


Ad-KLF5 and Ad-GFP were made as described previously [13]. Hela cells were infected with Adenoviral vector (Genechem Co., Ltd). To achieve RNAi-mediated depletion of Eppk1, we transfected cells with siRNA oligos targeting Eppk1 or negative controls (Genechem Co., Ltd). In this process, we also used the Lipofectamine 2000 reagents (Invitrogen; Thermo Fisher Scientific, Inc.) according to the operation instructions.

\section{Immunofluorescence staining.}

All cervical tissue samples were fixed by $4 \%$ paraformaldehyde solution, the fixed tissue was dehydrated by ethanol, transparent by xylene, embedded in paraffin, and the sections were $4 \mu \mathrm{m}$. The above samples were operated by indirect immunofluorescence method according to the instructions. rabbit anti-KLF5 (1:50, GTX103289, GeneTex) and mouse anti-Eppk1 (1:50, sc-87102, Santa) were incubated overnight at $4{ }^{\circ} \mathrm{C}$, Fluorescein labeled fluorescent secondary antibody, Rhodamine labeled fluorescent secondary antibody (KPL) and DAPI (Sigma) nuclear staining. Image Pro-Plus6.0 (Media Cybernetics, Inc, USA) Image analysis software analyzed the fluorescence intensity of Eppk1 and KLF5 expressions.

\section{Real-time PCR}

Total tissue RNA was extracted from the cervical tissues preserved in liquid nitrogen according to the instructions of Trizol kit. RNA was used as template, cDNA was synthesized by reverse transcription kit (Invitrogen, USA), and then cDNA was used as template for Real-time PCR. Glyceraldehyde-3-phosphate dehydrogenase (GAPDH) was used as internal reference. Apply Primer Premier software, design and downstream primers respectively: Eppk1 upstream Primer sequences for 5'- GGCCATGCCGATTTAAATGC3', The downstream primer sequences for 5'- CAAGCAAAGTCAGTCCAAGC-3'. The upstream primer sequence of GAPDH was 5'- CGTCCCGTAGACAAAATGGT-3'. The downstream primer sequence of GAPDH is 5'- GAGGTCAATGAAGGGGTCG-3'. Amplification reaction conditions: $95^{\circ} \mathrm{C}, 2 \mathrm{~min}, 95^{\circ} \mathrm{C}, 15 \mathrm{~s}, 72^{\circ} \mathrm{C}, 35 \mathrm{~s}$, 40 cycles. Formula $2^{-\triangle \triangle C t}$ (Ct as the circulating threshold) was used to calculate the mRNA expression level of the target gene, and the experiment was repeated 3 times for each group.

\section{Western blotting}

A RIPA kit (Beyotime Institute of Biotechnology) was operated to extract total proteins from treated cells, the total protein content was detected by the protein assay kit (Beyotime Biotechnology). SDS-PAGE was used to separate the protein samples with $8-12 \%$ separating gel and every protein sample added in the well was $40 \mu \mathrm{g}$. The separated proteins was resolved and electrophoretically moved to the PVDF membranes. After blocked with $5 \%$ defatted milk in TBST at room temperature for $1.5 \mathrm{~h}$, the blots were incubated with primary antibodies for a night at $4{ }^{\circ} \mathrm{C}$. The secondary antibody (1:2000) was incubated at room temperature, the membrane was washed, and the film was developed. The $\beta$-actin is the internal reference protein. The signals were detected with the ECL system (Pierce) and quantifed by scanning densitometry with the Image $\mathrm{J}$ analysis. The primary antibodies includes anti-KLF5 antibodies $(1: 1,000$, GTX103289, GeneTex), anti-Eppk1 antibodies (1:1,000, sc-87102, Santa), anti-p38 antibodies (1:1000, 14064-1-AP, Proteintech), p-p38 (1:1000, 14064-1-AP, Proteintech), AKT (1:1000, 10176-2-AP, Proteintech), 
p-AKT (1:1000, 66444-1-Ig, Proteintech), p-ERK1/2 (1:1000, cat. no.9101, Cell Signaling), ERK (1:1000, 16443-1-AP, Proteintech) and $\beta$-actin (1:5000, 20536-1-AP, Proteintech).

\section{Dual luciferase assays}

Lipofectamine-2000 was used to co-transfect pGL3-basic (negative control) vector, pGL3-Eppk1promoter, pGL3-Eppk1 promoter mutation, plasmid-expressed KLF5 or empty plasmid. The concentration of each plasmid was $0.5 \mu \mathrm{g} /$ well. Meanwhile, pRL- $\beta$-actin $10 \mathrm{ng} /$ well was co-transfected as internal reference. At $24 \mathrm{~h}$ after transfection, the cells were transfected with empty plasmid (pcDNA3.1) and plasmid-expressed KLF5 (pcDNA3.1-KLF5). At $48 \mathrm{~h}$ after transfection, luciferase activity was measured using the dualluciferase reporter assay system (Promega) The experiment was repeated three times, and the average value of the data was calculated.

\section{Chromatin immunoprecipitation assays}

The chromatin immunoprecipitation assay was performed using the HeLa cells following the protocol provided by Abcam (Cambridge, MA, USA). The diluted DNA- protein complex was incubated with an equal amount of anti-Eppk1 antibody or mouse IgG (Santa Cruz) overnight at $4^{\circ} \mathrm{C}$ in the presence of herring sperm DNA and protein A/G beads. Chromosomal DNA was purified and analyzed by RT-qPCR. The PCR primers for the Eppk1 gene promoter to amplify the KLF5-binding region were as follows: Forward: 5'TGGGGCCTGGTGGGGGGAAAG 3'; Reverse: 5'GGCCGGCCCCCTCTGACTCA 3'. There were three groups of samples: IgG (negative control), Input (positive control) and Ad- KLF5.

\section{Cell proliferation assay}

CCK-8 assay was used to detect the growth and proliferation of Hela cells. Hela vaccination in 96-well plates, $100 \mu \mathrm{l}$ per hole cell suspension, after waiting for cell adherent to group training, each group have 3 hole, cultivate 12 h, 24 h, 48 h, 72 h, 96 h after $10 \mu$ per hole to join CCK 8 solution, set a zero cell free hole, incubator to $4 \mathrm{~h}$ incubation, enzyme standard instrument determination at $450 \mathrm{~nm}$ OD value.

\section{Statistical analysis}

Data provided as mean \pm standard deviation $\left(\bar{x}_{ \pm} \pm s\right)$ was analyzed with SPSS13.0 (SPSS, Inc.) and GraphPad Prism 5.0 (GraphPad Software, Inc.). The pathological data collected from the samples was compared by using Chi-square test. Contrasts between two groups were performed through an unpaired two-tailed $t$ test. One-way ANOVA was used for multiple comparisons followed by the post hoc Turkey's test. $\mathrm{P}<0.05$ was considered statistically significant.

\section{Results}

Eppk1 expression is significantly increased in cervical cancer tissues and cell lines. 
To examined whether the levels of Eppk1 would be altered in cervical cancer, we performed RNA expression profiling using RNA-sequence analyses of human $\mathrm{CC}$ tissues $(\mathrm{n}=3)$ and normal cervical tissues $(n=3)$. As shown in Fig. 1a, mRNA level of Eppk1 was significantly upregulated in CC tissues. RTqPCR confirmed the increased expression of Eppk1 mRNA in CC tissues was by 8.7 times $(p<0.001)$ compared with the control (Fig. 1b). TCGA database further revealed that the level of Eppk1 in cervical squamous cell carcinoma was significantly increased $(p<0.05)$ (Fig. 1c). Moreover, we also found the correlation between Eppk1 and KLF5 in cervical cancer using the TCGA database $(R=0.18, P<0.01)$ (Fig. 1d). In subsequent western blotting assay in vitro, compared with normal cervical epidermal cells (HcerEpic), the protein levels of both Eppk1 and KLF5 were increased in the CC cell lines C33A, SiHa and Hela, especially in Hela (Fig. 1f).

In addition, we further performed immunofluorescence staining to examine co-expression of Eppk1 and KLF5 proteins in different pathologic cervical tissues ( 37 normal cervical tissues, cervical intraepithelial neoplasia (CIN) tissues (CIN I: 31 cases; CIN II-III: 47 cases) and 40 CC tissues) was detected by, as shown in Fig. 1e. The result showed that Eppk1 expression was significantly increased in CINII-III and SCC tissues accomplished with increased KLF5 (both $p<0.05$ and $p<0.01$ ). Moreover, Eppk1 was mainly located in the intercellular and cytoplasm, suggesting that it mainly plays the role of membrane receptor and skeleton. These data suggest that Eppk1 upregulation is involved in the tumorigenesis in cervical cancer, which is associated with increased KLF5 expression.

\section{KLF5 is a positive regulator of Eppk1 in CC cell.}

To further validate whether KLF5 is required for the expression of Eppk1 gene, we generated KLF5 overexpressing and knockdown Hela cells by infecting with adenoviruses encoding KLF5 (Ad-KLF5) or transfecting with siRNA against KLF5 (si-KLF5), respectively. Both RT-qPCR and Western blot results showed that the overexpression of KLF5 increased the expression of Eppk1 at transcription and translation levels (Fig. 2a, 2b). Conversely, knockdown of KLF5 suppresses Eppk1 expression in Hela cells (Fig. 2c, 2d). These results indicate that KLF5 plays a key role in the regulation of Eppk1 expression.

\section{KLF5 regulates Eppk1 expression by direct binding to the Eppk1 promoter.}

To demonstrate whether KIf 5 regulates the expression of Eppk1 by direct binding to its promoter, using the JASPAR CORE database, we performed a KLF5-binding motif analysis on the Eppk1 promoter, and identified one typical KLF5-binding site in the -1000 to +1 bp of the 5 'upstream promoter of Eppk1 gene (Fig. 3a). Subsequently, the Eppk1 promoter and its mutant were constructed and tested by the luciferase activity assay. The results showed that enforced KLF5 expression markedly increased the activity of the Eppk1 full-length promoter. Mutation of -295 to -285 bp region of the Eppk1 promoter significantly decreased activation by KLF5 $(P<0.01)$ (Fig. 3b), indicating that KLF5-binding site between -295 to 285 are critical for the KLF5-mediated transcriptional activation of the Eppk1 promoter. Moreover, chromatin immunoprecipitation (CHIP) assay further demonstrated that overexpression of KLF5 obviously increased its recruitment to the site (Fig. 3c). These results indicated that KLF5 activates Eppk1 transcription by directly binding the Eppk1 promoter. 


\section{KLF5 promotes CC cell proliferation dependent on Eppk1 expression.}

As essential role of transcription factor KLF5 in cell proliferation and tumorigenesis in CC, we determined whether Eppk1 expression can influence cell proliferation. As shown in Fig. 4a, Eppk1 expression was markedly attenuated in si-Eppk1-transfected HeLa cells relative to cells transfected with si-Con. Correspondingly, cell viability was significantly suppressed by CCK8 assay (Fig. 4b). Furthermore, enhanced KLF5 expression inducing Eppk1 expression and cell proliferation were both significantly decreased in si-Eppk1-transfected cells (Fig. 4c and 4d), suggesting that Eppk1 is required for cell proliferation in CC. These results suggested that KLF5 mediates Eppk1 expression promoting Hela cell proliferation.

\section{Eppk1 expression affects p38 signal pathway.}

Because Eppk1 is a component of EGFR, and Eppk1 expression has a positive correlation with EGFR in CC from TCGA data $(p<0.05, R=0.13$ ) (Fig. 5a), we further clarify the EGFR-related signaling pathways by which Eppk1 expression affects the proliferation of cervical cancer cells. As shown in Fig. 5b, the activation protein of $p$-p38, $p$-ERK1/2 and $p$-AKT were all significantly increased $(P<0.01)$ in Ad-KLF5infected Hela cells, whereas KLF5 expression-induced p-p38 activation was significantly decreased after si-Eppk1 transfection $(P<0.05)$, and p-AKT and p-ERK1/2 signaling pathways did not seem to be affected (Fig. 5b and 5c). In addition, we investigated the correlation between the expression of Eppk1 and clinicopathological parameters in CC. The results showed that the mRNA level of Eppk1 was correlated with HPV positive infection rate and tumor size $(P<0.05)$, but not with pathological grade and clinical stage, as shown in Table 1. Taken together, these data suggest that KLF5-mediated Eppk1 expression affecting p38 signaling, KLF5, Eppk1 and p38 co-regulate cell proliferation and the tumorigenesis in cervical cancer.

\section{Discussion}

The present study firstly demonstrates that Eppk1 plays a critical role in positively regulating AKT signaling mediated cell proliferation as well as Eppk1 as a direct target of KLF5. Specifically, we observed that knockdown of Eppk1 expression blocked KLF5-induced cell proliferation via p38 signaling pathway in Hela. Additionally, KLF5 and Eppk1 are co-expressed in human CC tissues and CC cell lines. Our results suggest that Eppk1 may be a potential therapeutic target for the treatment of cervical cancer.

Most plakins containing a plakin domain formed by up to nine spectrin repeats (SR1-SR9) and an SH3 domain are expressed in various organs and tissues [14], but detailed expression patterns has not been examined. Eppk1 as a member of plakin family, was expressed in various progenitors and developing and regenerating cells, especially in pancreatic cancer $[15,16]$, suggesting that Eppk 1 is a good marker for development of cancer cells. In this study, we focused on the expression pattarns of Eppk1 in cervical tissues and found that under-expression of Eppk1 is in normal cervical tissues and is slightly increased in CIN囚tissues. However, it was significantly increased in severe pathological changes of cervical tissues,

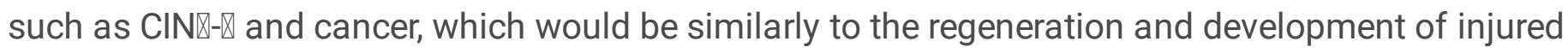


tissues or cancer associated with upregulation of Eppk1. It is hypothesized that some cancers represent an aberrant recapitulation of normal development, even that further deduction that some cancers are originated from adult tissue stem cell [17]. Moreover, expression of Eppk1 was positive correlation with tumor size in CC tissues (Table 1). Therefore, study of Eppk1 expression might shed lights on initiation and development of cervical cancer. In addition, Marrero-rodriguez et al. found that KLF5 showed a gradual up-regulation trend in normal tissues, low-grade intraepithelial lesions, intraepithelial squamous lesions and CC samples, indicating that the up-regulation of KLF5 expression plays a certain role in the occurrence and development of cervical cancer [18]. We also demonstrated that the co-expression pattern between KLF5 and Eppk1 as well as Eppk1 is a target of KLF5 in CC cells, suggesting that inhibition of KLF5 expression by pharmacologic treatment (such as ML264) may be beneficial for treatment of CC.

It is well known that elevated levels of the epidermal growth factor receptor (EGFR), a growth-factorreceptor tyrosine kinase, and/or its cognate ligands have been identified as a common component of multiple cancer types and promote solid tumor growth [19]. When EGFR is activated by growth factors, it mainly regulates cell proliferation and other processes through downstream signaling pathways [20]. EGFR activation-associated downstream signaling pathway mainly includes ERK [21], p38 [22] and AKT [23] signalings, which need to explored the potential functional mechanism by which Eppk1 mediates cell proliferation of CC. Furthermore, KLF5 is widely expressed in different tissues and plays a higher role in cell proliferation than in cell differentiation [24]. It has been reported that KLF5 is a positive regulator of the RAS/MAPK signaling pathways, and the RAS/MAPK pathway is affected by KLF5-mediated EGFR activation $[25,26]$. Our previous study also revealed the p38 signaling pathway mediated the effects of KLF5 on CC cell proliferation [12]. In addition, Eppk1-positive cells express PCNA (a proliferation marker), which plays an important role in transient amplification of cells [27], and Blagoev et al found that plakin was identified as one of the proteins binding to EGF receptor using proteomics, suggesting that Eppk1 is involved in EGFR-related proliferation signal transduction in tumor cells [28]. In our experiments, we demonstrated that the expression of Eppk1 is positively correlation with EGFR from TCGA database, simultaneously inhibition of p38 phosphorylation by Eppk1 knockdown indicate that KLF5 and Eppk1 coregulate p38 signaling pathway during cell proliferation, indicating that cell proliferation of CC partly dependent on Eppk1 expression via p38 signaling pathway in context of KLF5 expression, but not the ERK or AKT signaling pathway. Therefore, inhibition of Eppk1 or its downstream p38 signaling pathway may be may exert benefcial efect for attenuation of CC initiation and development.

\section{Conclusions}

Overall, the present study showed that KLF5-mediated upregulation of Eppk1 can activate the p38 signaling pathway to promote the proliferation of CC cells. There are some limits in this study: Firstly, the role of Eppk1 in CC cell growth should be verified in vivo; Secondly, some other biological functions of Eppk1 in CC cells still need to be assessed. Last, Eppk1 deficiency in CC model causing a phenotype of tumor growth inhibition is also critical. However, this work excites attention on the role of Eppk1 in CC and gives the direction in future research, contributing to a novel molecular target for the clinical diagnosis and treatment of cervical cancer. 


\section{Abbreviations}

Eppk1: Epiplakin1; HPV: human papillomavirus; KLF5: Kruppel-like factors 5; CIN: cervical intraepithelial neoplasia; SCC: squamous cell carcinomas; FIGO: Federation of Gynaecology and Obstetrics; HcerEpic: Human normal cervical epithelial cell; DAPI: 2-(4-Amidinophenyl)-6-indolecarbamidine dihydrochloride; GAPDH: Glyceraldehyde-3-phosphate dehydrogenase; $\mathrm{Ct}$ : the circulating threshold; Ad-KLF5: adenoviruses encoding KLF5; si-KLF5: siRNA against KLF5; CHIP: chromatin immunoprecipitation; EGFR: the epidermal growth factor receptor; ERK: extracellular signal-regulated kinase; MAPK: mitogen-activated protein kinase; PCNA: Proliferating Cell Nuclear Antigen

\section{Declarations}

\section{Acknowledgements}

We thank Quan Chang, Jin-jin Zhang for excellent technical assistance, Xiao Liu, Na Hua, Guo-Hua Li for clinical assistance.

\section{Author contributions}

$\mathrm{ZP}, \mathrm{QC}, \mathrm{JJZ}$ and DM performed most experiments and analyzed data, $\mathrm{XL}, \mathrm{NH}$ and $\mathrm{GHL}$ performed and supervised clinical experiments. ZP and DM conceived the study, supervised experiments and wrote the manuscript. All authors read and approved the final manuscript.

\section{Funding}

This work is supported by the National Natural Science Foundation of China (No. 81541149), Natural Science Foundation of Hebei Province (No. H2016209095), 2018 Hebei Provincial Medical Science Research Major Projects (No. 20180729).

\section{Availability of data and materials}

All data generated or analysed during this study are included in this published article.

\section{Ethics approval}

Cervical cancer (CC) specimens were obtained from patients undergoing surgical resection upon informed consent and approval by Ethical Committee of the North China University of Science and Technology (approval number: 15070).

\section{Consent for publication}

Not Applicable.

\section{Competing interests}


The authors declare that they have no competing interests.

\section{Author details}

${ }^{1}$ School of Public Health, North China University of Science and Technology, 21 Bohai Avenue, Caofeidian New City, Tangshan 063210, China; ${ }^{2}$ Department of Infection Control, the Fourth Hospital of Hebei Medical Univertisy, No.12 Jian Kang Rd. Shi Jiazhuang, 050011, China; ${ }^{3}$ Tangshan Customs, People's Republic of China, Tangshan, 063200, China; ${ }^{4}$ Department of Oncology, the Second Hospital of Tangshan, Tangshan, 063000, China.

\section{References}

1. Siegel RL, Miller KD, Jemal A. Cancer statistics. 2015. CA: a cancer journal for clinicians. 2015;65(1):5-29.

2. Verma J, Monk BJ, Wolfson AH. New strategies for multi-modality therapy in treating locally advanced cervix cancer. Semin Radiat Oncol. 2016;26(4):344-8.

3. Bosch FX, Manos MM. Prevalence of human papillomavirus in cervical cancer: A worldwide perspective. International biological study on cervical cancer (IBSCC) Study Group. J Natl Cancer Inst. 1995;87(11):796-802.

4. Fujiwara S, Takeo N, Otani Y, et al. Epiplakin, a novel member of the Plakin family originally identified as a 450-kDa human epidermal autoantigen. Structure and tissue localization. J Biol Chem. 2001;276(16):13340-7.

5. Takeo N, Wang W, Matsuo N, et al. Structure and heterogeneity of the human gene for epiplakin (EPPK1). J Invest Dermatol. 2003;121(5):1224-36.

6. Blagoev B, Kratchmarova I, Ong SE, et al. A proteomics strategy to elucidate functional proteinprotein interactions applied to EGF signaling. Nat Biotechnol. 2003;21(3):315-8.

7. Shen T, Lu Y, Zhang Q. High squalene epoxidase in tumors predicts worse survival in patients with hepatocellular carcinoma: integrated bioinformatic analysis on NAFLD and HCC. Cancer Control. 2020;27(1):1-9.

8. Lee H, Kim K, Woo J, et al. Quantitative proteomic analysis identifies AHNAK (neuroblast differentiation-associated protein AHNAK) as a novel candidate biomarker for bladder urothelial carcinoma diagnosis by liquid-based cytology. Mol Cell Proteomics. 2018;17(9):1788-802.

9. Guo X, Hao Y, Kamilijiang M, et al. Potential predictive plasma biomarkers for cervical cancer by 2DDIGE proteomics and Ingenuity Pathway Analysis. Tumor Biol. 36(3):1711-1720.

10. Smith A, Poole L, Dhanwada K, et al. Identification of candidate biomarkers and cancer genes AHNAK2 and EPPK1 in pancreatic cancer. BJMMR. 2016;18(8):2231-0614.

11. Dong JT, Chen C. Essential role of KLF5 transcription factor in cell proliferation and differentiation and its implications for human diseases. Cell Mol Life Sci. 2009;66(16):2691-706. 
12. Ma D, Chang LY, Zhao S, et al. KLF5 promotes cervical cancer proliferation, migration and invasion in a manner partly dependent on TNFRSF11a expression. Sci Rep. 2017;7(1):15683.

13. Shi JH, Zheng B, Chen S, et al. Retinoic acid receptor a mediates all-trans-retinoic acid-induced Klf4 gene expression by regulating klf4 promoter activity in vascular smooth muscle cells. J Biol Chem. 2012;287(14):10799-811.

14. Ortega E, Manso JA, Buey RM, et al. The structure of the plakin domain of plectin reveals an extended rod-like shape. J Biol Chem. 2016;291(36):18643-62.

15. Sawamura D, Li K, Chu ML, et al. Human bullous pemphigoid antigen (BPAG1). Amino acid sequences deduced from cloned cDNAs predict biologically important peptide segments and protein domains. J Biol Chem. 1991;266(27):17784-90.

16. Yoshida T, Shiraki N, Baba H, et al. Expression patterns of epiplakin1 in pancreas, pancreatic cancer and regenerating pancreas. Genes Cells. 2008;13(7):667-78.

17. Dunne RF, Hezel AF. Genetics and biology of pancreatic ductal adenocarcinoma. Genes Dev. 2015;29(4):595-608.

18. Marrero-Rodrgíuez D, Taniguchi-Ponciano K, Jimenez-Vega F, et al. Krüppel-like factor 5 as potential molecular marker in cervical cancer and the KLF family profile expression. Tumor Biol. 2014;35(11):11399-407.

19. Nicholson RI, Gee JM, Harper ME. EGFR and cancer prognosis. Eur J Cancer. 2001;37(suppl 4):9-15.

20. Ciardiello F. A novel approach in the treatment of cancer: targeting the epidermal growth factor receptor. Clin Cancer Res. 2001;7(10):2958-70.

21. Ohm AM, Affandi T, Reyland ME. EGF receptor and PKC $\delta$ kinase activate DNA damage-induced prosurvival and pro-apoptotic signaling via biphasic activation of ERK and MSK1 kinases. J Biol Chem. 2019;294(12):4488-97.

22. XieZB, Zhang YF, Jin C, et al. LRG-1 promotes pancreatic cancer growth and metastasis via modulation of the EGFR/p38 signaling. J Exp Clin Cancer Res. 2019;38(1):75.

23. Cao Q, Wang N, Ren L, et al. MiR-125a-5p post-transcriptionally suppresses GALNT7 to inhibit proliferation and invasion in cervical cancer cells via the EGFR/PI3K/AKT pathway. Cancer Cell Int. 2020;20:117.

24. McConnell BB, Yang VW. Mammalian Krüppel-like factors in health and diseases. Physiol Rev. 2010;90(4):1337-81.

25. Nandan MO, Yoon HS, Zhao W, et al. Krüppel-like factor 5 mediates the transforming activity of oncogenic H-Ras. Oncogene. 2004;23(19):3404-13.

26. Yang $Y$, Goldstein BG, Nakagawa $H$, et al. Krüppel-like factor 5 activates MEK/ERK signaling via EGFR in primary squamous epithelial cells. FASEB J. 2007;21(2):543-50.

27. Matsuo A, Yoshida T, Yasukawa T, et al. Epiplakin1 is expressed in the cholangiocyte lineage cells in normal liver and adult progenitor cells in injured liver. Gene Expr Patterns. 2011;11(3-4):255-62. 
28. Lagoev B, Kratchmarova I, Ong SE, et al. A proteomics strategy to elucidate functional protein-protein interactions applied to EGF signaling. Nat Biotechnol. 2003;21(3):315-8.

\section{Tables}

Table 1 The relationship between expression of Eppk1 mRNA and pathological characteristics of CC

\begin{tabular}{|c|c|c|c|c|c|}
\hline \multirow[t]{2}{*}{ Parameter } & $\mathrm{N}$ & Eppk1 low expression & Eppk1 high expression & \multirow[t]{2}{*}{$\chi^{2}$} & \multirow[t]{2}{*}{$P$} \\
\hline & 40 & $\mathrm{~N}$ & 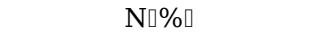 & & \\
\hline HPV16/18type & & & & 4.42 & 0.035 \\
\hline positive & 29 & $21 \square 72.4 \square$ & $8 \square 27.6 \square$ & & \\
\hline negative & 11 & $4 \square 36.4 \square$ & $7 \square 63.6 \square$ & & \\
\hline Tumor size $\llbracket \mathrm{cm} \rrbracket$ & & & & 6.07 & 0.014 \\
\hline$\leq 4$ & 14 & $10 \square 71.4 \square$ & $4 \square 28.6 \square$ & & \\
\hline$\square 4$ & 26 & $8 \square 30.8 \square$ & $18 \square 69.2 \square$ & & \\
\hline ogical grading & & & & 2.13 & 0.145 \\
\hline nd medium differentiation & 13 & $8 \square 61.5 \square$ & $5 \square 38.5 \square$ & & \\
\hline soorly differentiated & & & & & \\
\hline clinical stages & 27 & $10 \square 37.0 \square$ & $17 \square 63.0 \square$ & & \\
\hline प & & & & 1.32 & 0.251 \\
\hline प & 23 & $15 \square 65.2 \square$ & $8 \square 34.8 \square$ & & \\
\hline & 17 & $8 \square 47.1 \square$ & $9 \square 52.9 \square$ & & \\
\hline
\end{tabular}

\section{Figures}


a

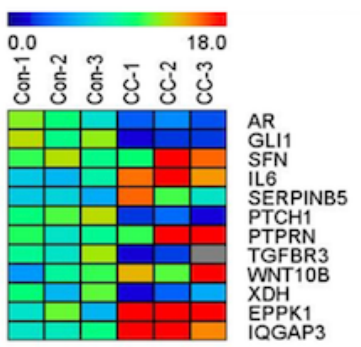

e

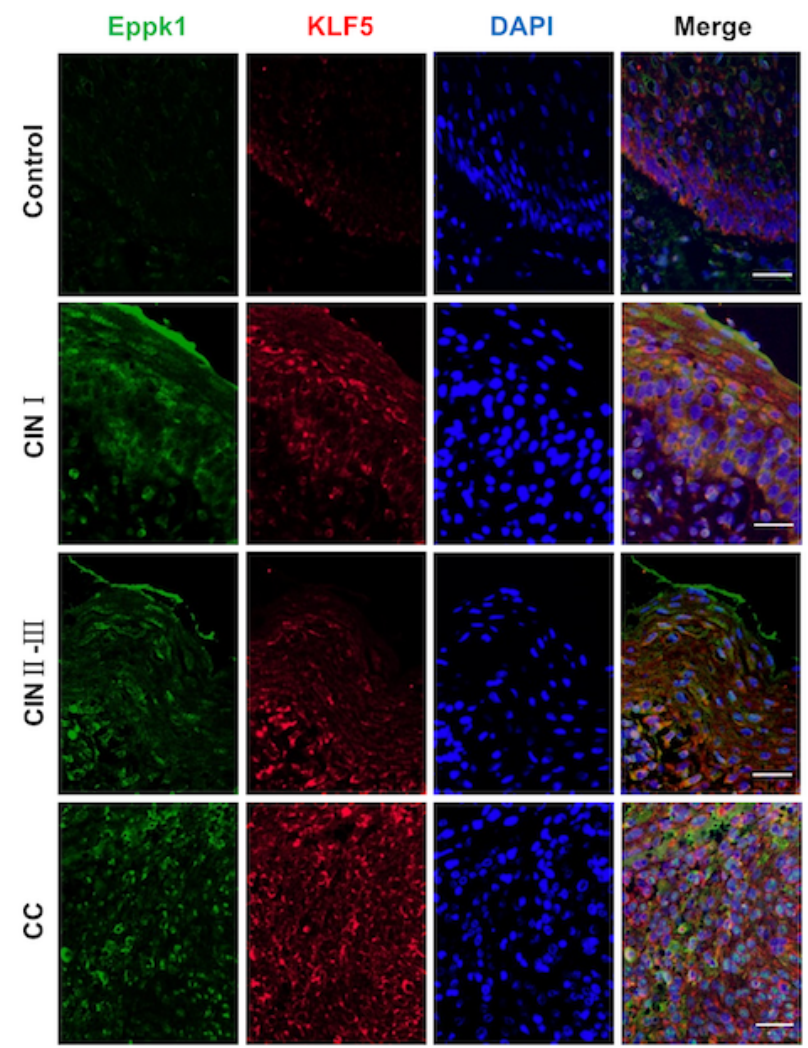

C
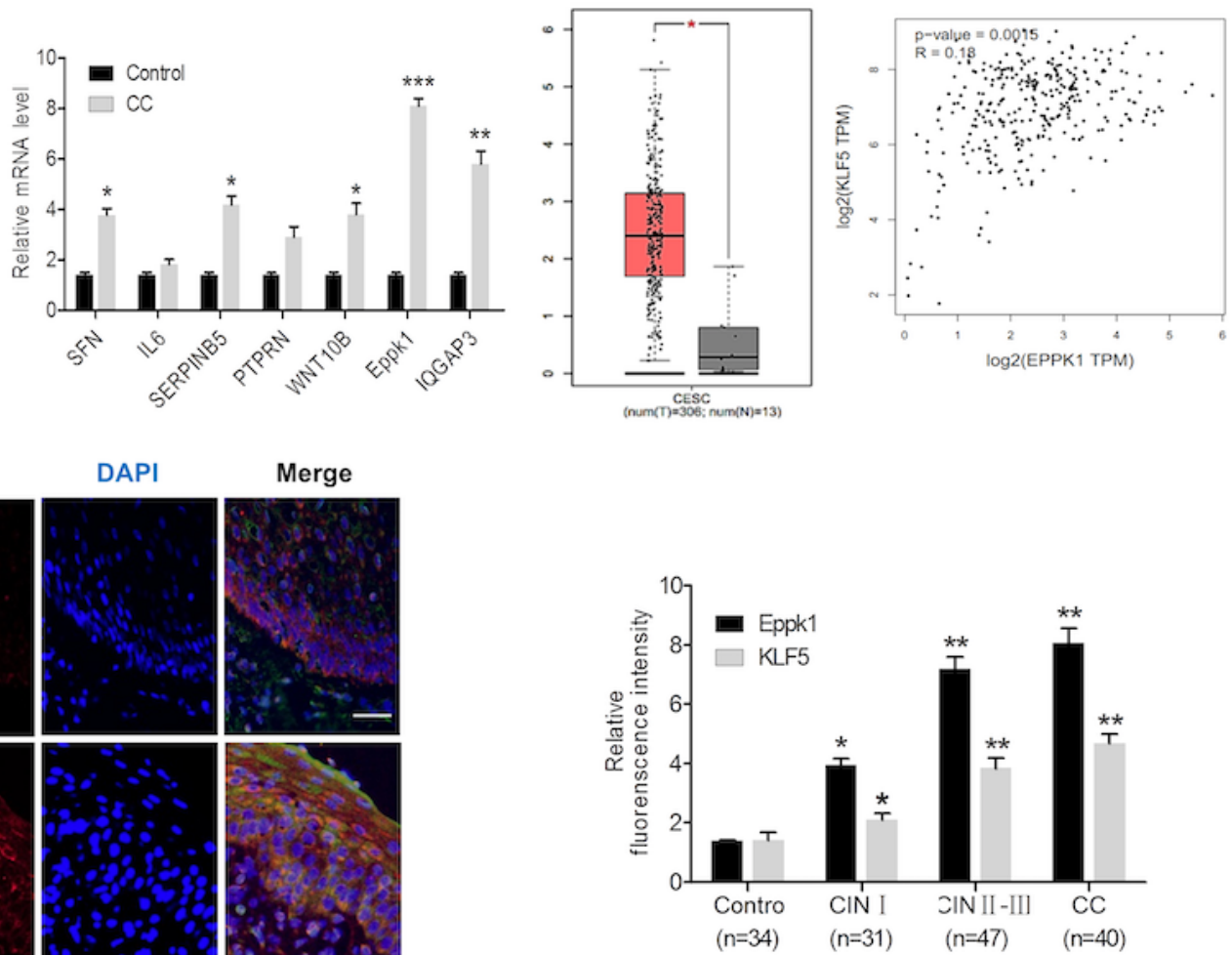

f

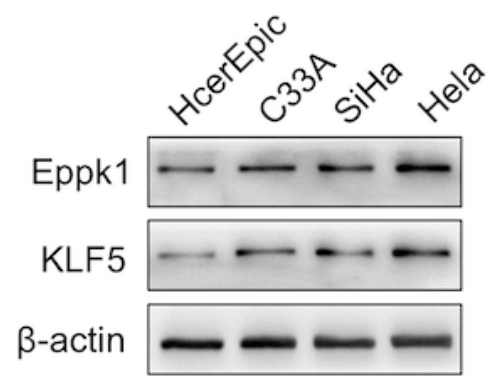

\section{Figure 1}

Eppk1 expression is significantly increased in cervical cancer tissues and cell lines. a Heatmap illustrates a subset of the differentially expressed mRNAs detected in 3 cervical cancer tissues and 3 normal tissues (control) by microarray analysis. b Seven changed genes were validated by qRT-PCR. ${ }^{*} *$ P $<0.001$ vs control. c,d mRNA expression of Eppk1 (c) and correlation analysis between mRNA expression of Eppk1 and KLF5 (d) in cervical squamous cell carcinoma (CSCC) from the Cancer Genome Atlas (TCGA) database. e Immunofluorescence staining of KLF5 (red) and Eppk1(green) in CC tissues ( $n=40)$, cervical intraepithelial neoplasia (CIN) tissues (CIN I: 31 cases; CIN II-III: 47 cases) and control ( $n=37)$. Scale bars $=100 \mu \mathrm{m}$. Right: Statistical analysis of fluorescence intensity. ${ }^{*} \mathrm{P}<0.05$ and ${ }^{*} \mathrm{P}<0.01 \mathrm{vs}$. control. $\mathrm{f}$ Expression level of Eppk1 and KLF5 protein in C33A, HeLa and SiHa cells. 


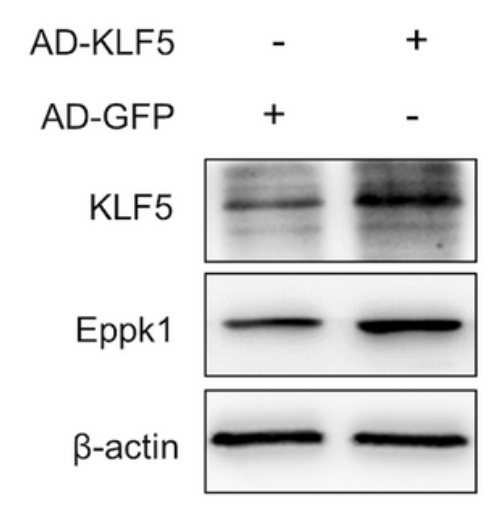

C

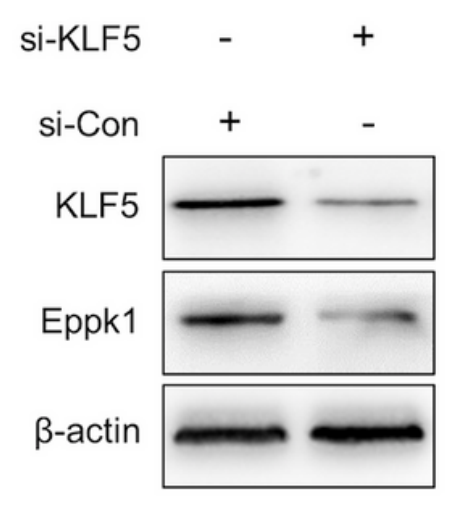

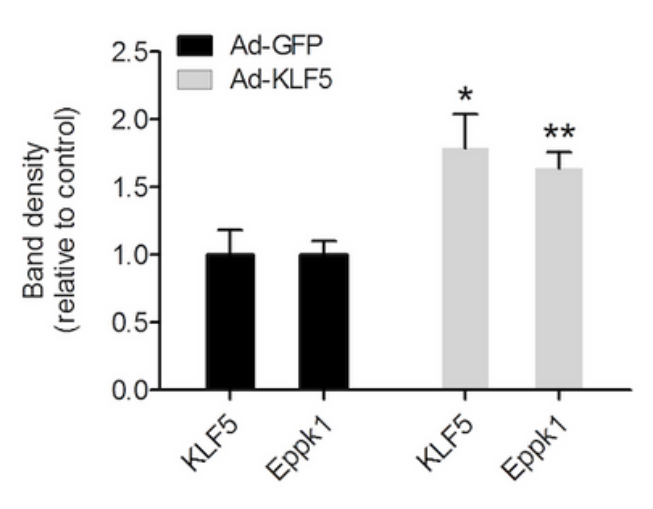

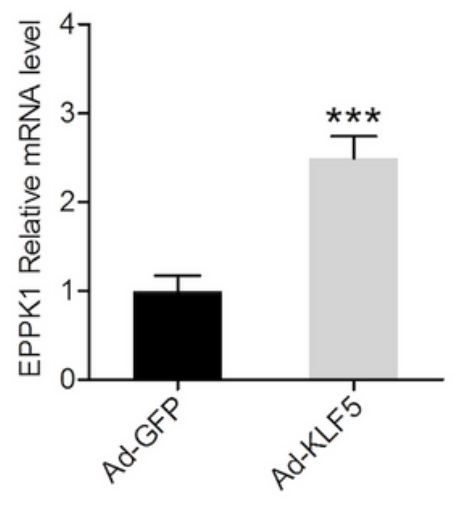

d

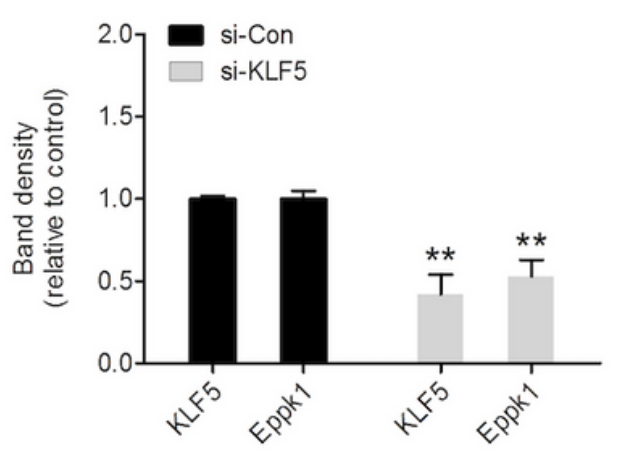

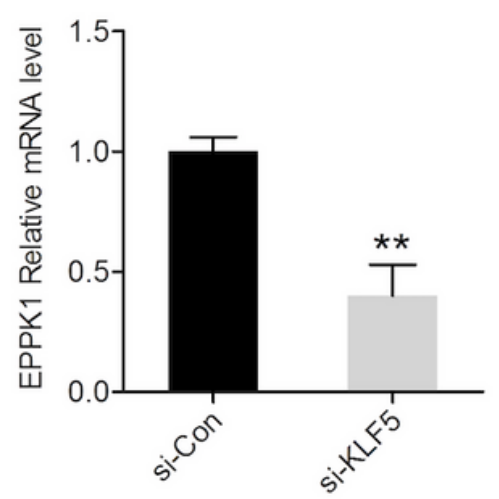

Figure 2

KLF5 is a positive regulator of Eppk1 in Hela cell. a,b HeLa cells were infected with adenoviruses expressing green fluorescent protein (Ad-GFP) or KLF5 (Ad-KLF5) for $24 \mathrm{~h} \mathrm{(10} \mathrm{ng/mL).} \mathrm{KLF5} \mathrm{and} \mathrm{Eppk1}$ protein and mRNA expression were analyzed by western blot (a) and qRT-PCR (b), respectively. ${ }^{*}<0.05$ and ${ }^{*} \mathrm{P}<0.01$ vs. the Ad-GFP. c,d HeLa cells were transfected with non-specific short interfering RNA (siCon) or KLF5-specific siRNA (si-KLF5) for $24 \mathrm{~h}$. KLF5 and Eppk1 protein and mRNA expression were analysed by western blot (c) and qRT-PCR (d), respectively. ${ }^{*} P<0.05$ vs. the si-Con. 
a

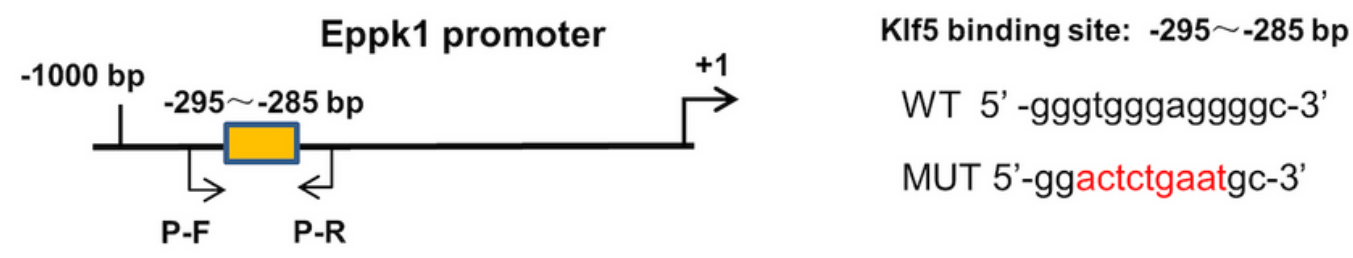

b
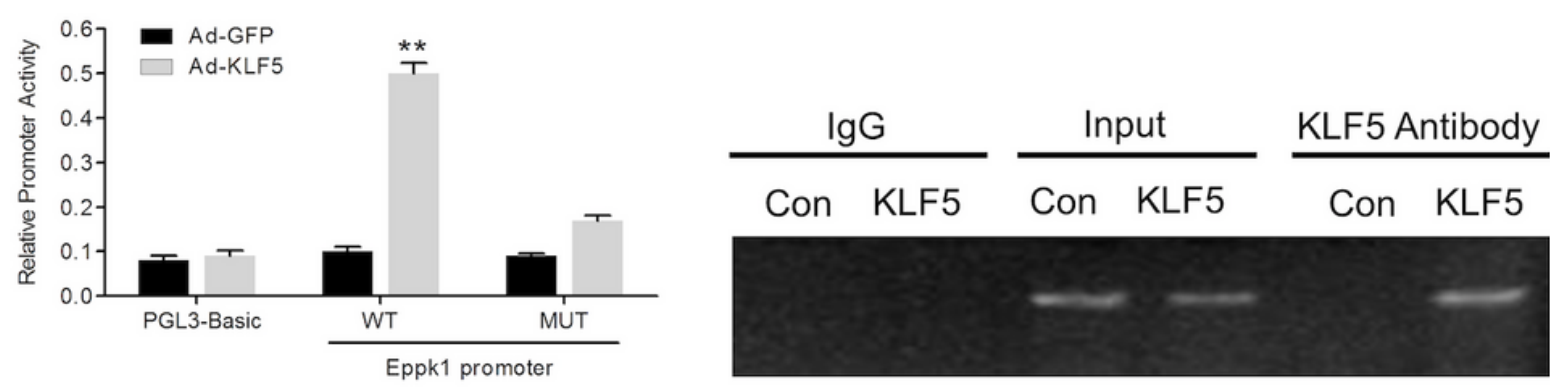

\section{Figure 3}

KLF5 regulates Eppk1 expression by direct binding to Eppk1 promoter. a A schematic map of the -1000 to +1 bp region of the Eppk1 promoter showing a KLF5-binding site at -295 to -285 bp region. b A luciferase reporter controlled by the Eppk1 promoter or the Eppk1 promoter with mutation in KLF5-binding site was transfected into Hela cells along with increasing amounts of KLF5 expression plasmid (pcDNA3.1-KLF5). pGL3-Basic was used as the negative control. Luciferase activity was measured using the dual luciferase reporter assay system. Data represent the relative Eppk1 promoter activity normalized to $\mathrm{pRL}$-TK activity. ${ }^{*} \mathrm{P}<0.05$ vs. pcDNA3.1. c Hela cells were infected with or without Ad-KLF5 for $24 \mathrm{~h}$. ChIP assay was then performed with antibody against Klf5. Nonimmune IgG was used as negative control. Immunoprecipitated DNA was amplified by PCR using the primers indicated as in (a). 

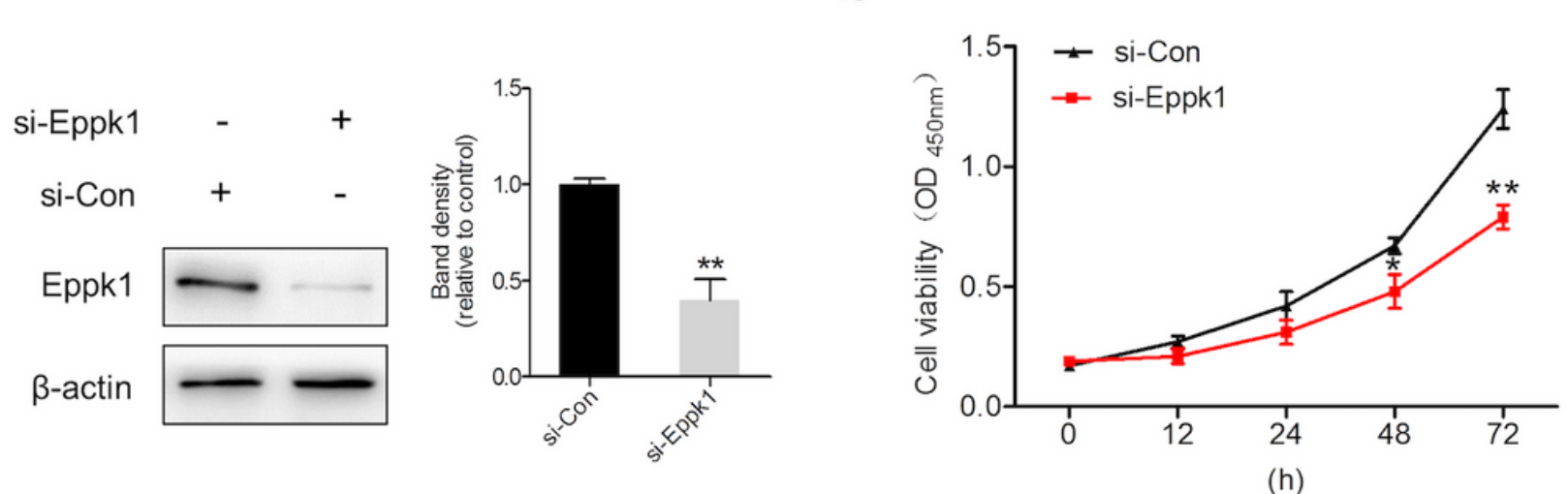

(h)
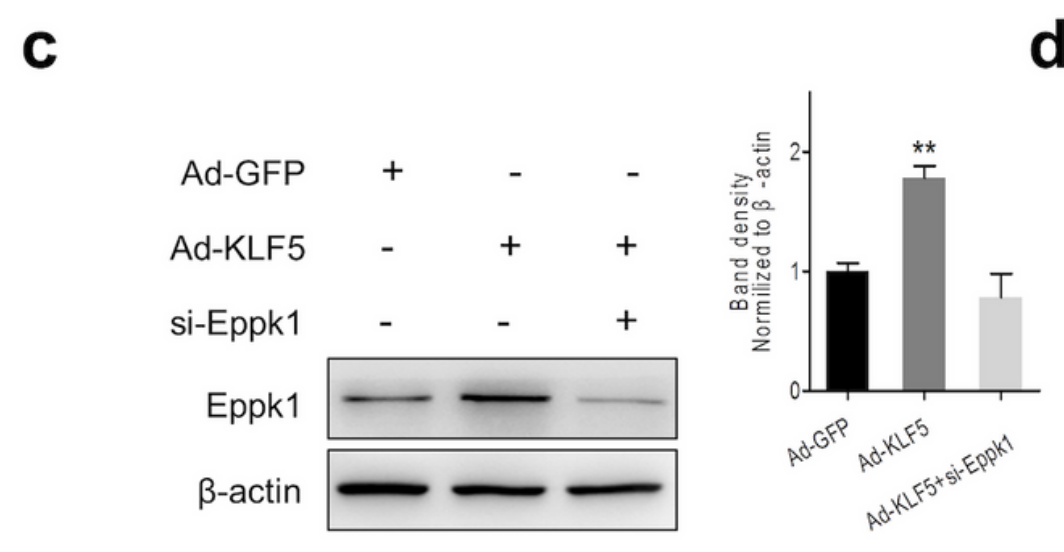

d

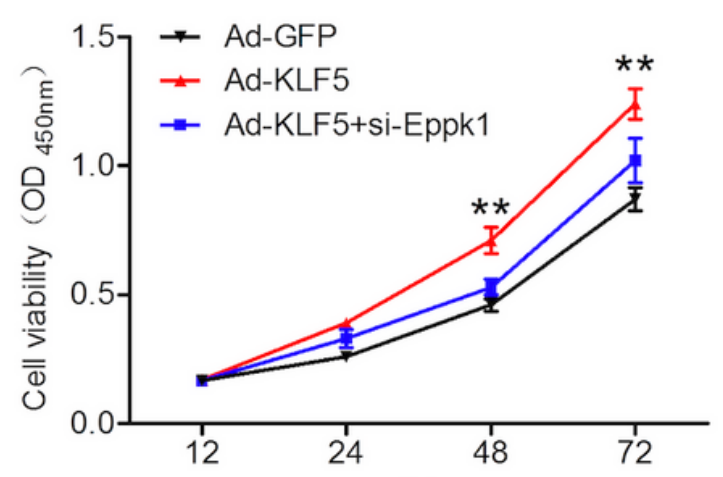

(h)

\section{Figure 4}

Eppk1 lies downstream of KLF5 and promotes Hela cell proliferation. a HeLa cells were transfected with si-Con or Eppk1-specific siRNA (si-Eppk1) for $24 \mathrm{~h}$. Eppk1 protein expression was analysed by western blot. $\beta$-actin was used as the loading control. Right: band intensities were measured and normalized to $\beta$ actin $(n=3)$. Data represent the mean \pm SD. ${ }^{*} P<<0.01$ vs. si-Con. b Knockdown of Eppk1 significantly affected HeLa cell growth compared to transfection with si-Con at different indicated time points by a CCK-8 assay $(n=3)$. Data represent the means \pm SD. ${ }^{*} P<0.05$ and ${ }^{*} P<0.01$ vs. the si-Con. $c$ Ad-GFP and Ad-KLF5-infected HeLa cells as well as Ad-KLF5-infected HeLa cells were transfected with si-Eppk1 for 36 h. Expression of Eppk1 was confirmed by western blot. Right: band intensities were measured and normalized to $\beta$-actin. Data represent the mean \pm SD. ${ }^{*} P<0.01$ vs. Ad-GFP. $d$ CCK- 8 assay for cell proliferation in Hela cells were treated as in (b). Data represent the means $\pm S D$. ${ }^{\star}{ }^{*} P<0.05$ vs. Ad-GFP. 

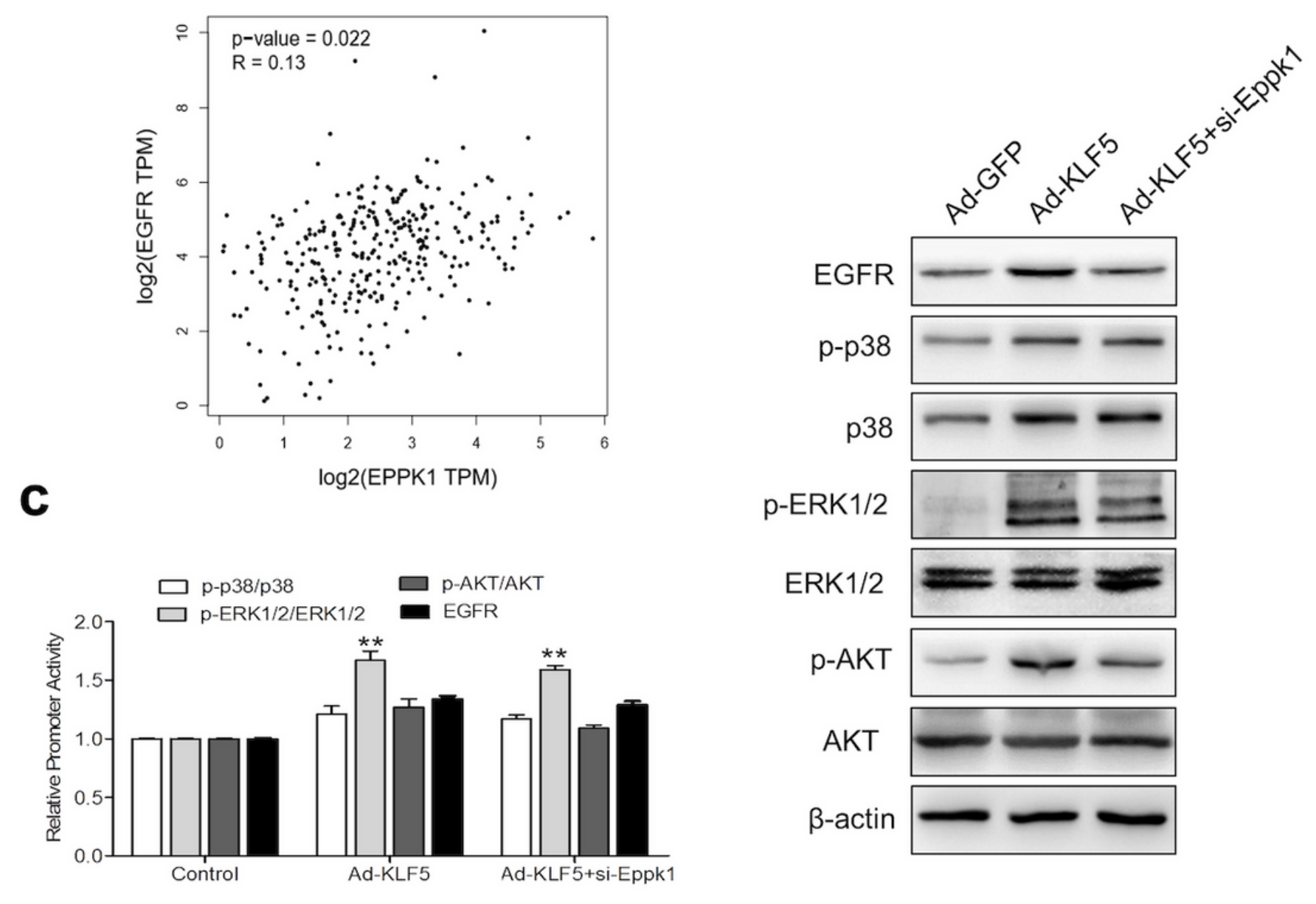

\section{Figure 5}

Eppk1 affects EGFR expression and p38 signal pathway. a Correlation analysis between mRNA level of Eppk1 and EGFR in CSCC tissues from TCGA database. b Ad-GFP and Ad-KLF5-infected HeLa cells as well as Ad-KLF5-infected HeLa cells were transfected with si-Eppk1 for $36 \mathrm{~h}$. Western blot was used to determine the activation of AKT, ERK1/2 and p38 signaling. $c$ band intensities were measured and normalized to $\beta$-actin or total AKT, ERK1/2 and P38 $(n=3)$. ${ }^{*}<0.05$ and ${ }^{* * P}<0.01$ vs. control, $\# P<0.05$ vs. Ad-GFP. 